\title{
The Long Non-Coding RNA SNHG1 Attenuates Cell Apoptosis by Regulating miR-195 and BCL2-Like Protein 2 in Human Cardiomyocytes
}

\author{
Ning Zhang ${ }^{\mathrm{a}} \quad$ Xin Meng ${ }^{\mathrm{a}} \quad$ Lijun Mei $^{\mathrm{b}} \quad$ Jian Hu $\mathrm{Hu}^{\mathrm{a}} \quad$ Chedong Zhao ${ }^{\mathrm{a}} \quad$ Wei Chen ${ }^{\mathrm{a}}$ \\ aClinical Laboratory, The First Affiliated Hospital of Xi'an Jiaotong University, Xi'an, ${ }^{b}$ Department of \\ Blood Transfusion, The Ankang Central Hospital, Ankang, China
}

\section{Key Words}

Cardiomyocyte $\bullet$ Hydrogen peroxide $・$ Apoptosis $・ \mathrm{SNHG} 1 \cdot \mathrm{miR}-195 \cdot \mathrm{BCL} 2 \mathrm{~L} 2$

\begin{abstract}
Background/Aims: Long non-coding RNAs (IncRNAs) are theorized to play key roles in the development of heart diseases. However, the role of IncRNAs in cardiomyocyte apoptosis is largely unknown. The present study examined the role of IncRNA SNHG1 in the human cardiomyocytes (HCMs) apoptosis and explored the underlying molecular mechanisms. Methods: SNHG1, miR-195 and mRNA expression was detected by qRT-PCR; protein level was determined by western blot; cell viability was detected by MTT assay; cell apoptosis was evaluated by flow cytometry and caspase-3 activity assay; the interaction between SNHG1 and miR195 was examined by using luciferase reporter assay. Results: Hydrogen peroxide $\left(\mathrm{H}_{2} \mathrm{O}_{2}\right)$ treatment significantly suppressed cell viability and increased cell apoptotic rate and caspase-3 activity in HCMs. Overexpression of SNHG1 attenuated the effects of $\mathrm{H}_{2} \mathrm{O}_{2}$ on $\mathrm{HCMs}$ viability and apoptosis; while SNHG1 exerted the opposite effects. SNHG1 was found to sponge miR-195 and suppress the expression of miR-195 in HCMs. Overexpression of miR-195 suppressed cell viability and induced apoptosis in $\mathrm{HCMs}$, and miR-195 was found to negatively regulate the expression of $B C L-2$ like protein 2 (BCL2L2) via targeting its $3^{\prime}$ untranslated region. Overexpression of $B C L 2 L 2$ partially reversed the effects of miR-195 overexpression on cell viability and cell apoptosis of HCMs. MiR-195 overexpression or BCL2L2 knockdown attenuated the effects of SNHG1 overexpression on cell viability, cell apoptosis and protein levels of cleaved caspase-3, cleaved caspase- 9 and $\mathrm{Bax}$ in $\mathrm{H}_{2} \mathrm{O}_{2}$-treated HCMs. Conclusion: Our results suggest a novel SNHG1/miR-195/BCL2L2 axis in the regulation of cardiomyocyte apoptosis. Modulation of SNHG1 may represent a novel strategy to treat cardiomyocyte apoptosis-related heart diseases.
\end{abstract}

\begin{tabular}{ll}
\hline Wei Chen & Clinical Laboratory, The First Affiliated Hospital of Xi'an Jiaotong University \\
& Xi'an, Shaanxi Province, 710061 (China) \\
& Tel. +86-13709269757, E-Mail chenwei808@mail.xjtu.edu.cn
\end{tabular}

\section{KARGER}




\section{Introduction}

Ischemic heart disease is the leading cause of death worldwide and is the most common consequence of coronary artery disease [1]. Cardiomyocyte death is an important factor in the development of heart diseases, such as heart failure, stroke and myocardial infarction [2]. Although occluded human coronary reperfusion is effective in reducing overall mortality, studies have shown that the restoration of blood flow through previous ischemic myocardium can result in additional reperfusion injury, including cell death and cardiomyocyte dysfunction [3-5]. Ischemia/reperfusion-induced cell injury is complex and involves a variety of signaling pathways. Recent studies have shown that apoptosis plays a key role in the cardiac damage caused by ischemia/reperfusion $[6,7]$. Unfortunately, the molecular mechanism involved in regulating apoptosis of cardiomyocytes remains unclear.

Recently, the long non-coding RNAs (IncRNAs) are theorized to play important roles in the process of ischemic heart disease [8]. LncRNAs are a class of non-protein-coding transcripts longer than 200 nucleotides, and play key roles in various biological processes such as DNA damage, microRNA (miRNA) silencing, programmed cell death, development, inflammation, and tumor progression $[9,10]$. The role of lncRNAs in the process of ischemic heart disease has also been revealed in several studies. The cardia apoptosis-related lncRNA was found to suppress mitochondrial fission and apoptosis by targeting miR-539 and prohibitin 2 in the cardiomyocytes [11]. The IncRNA, autophagy promoting factor functioned to regulate autophagic cell death via targeting miR-188-3p and ATG7 [12]. Studies from Zhang et al., suggested that lncRNA, myosin heavy chain associated RNA transcripts (MHRT) is a protective factor for cardiomyocytes and the plasma concentration of MHRT may serve as a biomarker for myocardial infarction diagnosis in acute myocardial infarction [13]. The lncRNA, small nucleolar RNA host gene 1 (SNHG1) is a newly-identified lncRNA and has been found to modulate cancer progression in various types of cancers via different molecular mechanisms, and in vitro studies revealed that SNGH1 increased cell viability and inhibited cell apoptosis in the cancer cells [14-17]. However, whether SNHG1 involves in the cellular behaviors of cardiomyocytes is unknown.

The miRNAs are small non-coding RNAs that post-transcriptionally regulate gene expression by targeting its 3' untranslated region (3'UTR). The role of miRNAs in the regulation of cardiomyocyte apoptosis has been well documented in various studies, and these miRNAs include miR-150, miR-19a, miR-200c, miR-1 and so on [18-21].

In this study, we demonstrated the regulatory role of SNHG1 in cardiomyocyte apoptosis, and we found that SNHG1 functioned as a competing endogenous RNA (ceRNA) to regulate the expression of miR-195 in the human cardiomyocytes (HCMs). In addition, SNHG1 also positively regulated the expression BCL-2 like protein 2 (BCL2L2), which is a target of miR-195. Our results suggest a novel SNHG1/miR-195/BCL2L2 axis in the regulation of cardiomyocyte apoptosis.

\section{Materials and Methods}

\section{Cell culture}

The HCMs isolated from the ventricles of the adult heart were purchased from PromoCell (Heidelberg, Germany). Cells were grown in in a monolayer to $80 \%$ confluence and then subcultured in the Ready-to-Use Myocyte Growth Medium (Promocell). HCMs at passages three to seven were used for experiments. The HEK293 cells were purchased from ATCC (Manassas, USA) and cells were cultured in the DMEM medium (Thermo Fisher Scientific, Waltham, USA) supplemented with 10\% fetal bovine serum (FBS; Gibco, Thermo Fisher Scientific), and were kept in a humidified incubator with $5 \% \mathrm{CO}_{2}$ at $37{ }^{\circ} \mathrm{C}$. 


\section{Cellular Physiology Cell Physiol Biochem 2018;50:1029-1040 \begin{tabular}{ll|l} 
and $10.1159 / 000494514$ & $\begin{array}{l}\text { O } 2018 \text { The Author(s). Published by S. Karger AG, Basel } \\
\text { www.karger.com/cpb }\end{array}$ \\
\hline and Biochemistry
\end{tabular}}

Zhang et al.: SNHG1 and Cardiomyocyte Apoptosis

Plasmids, miRNAs, siRNAs, transfection and $\mathrm{H}_{2} \mathrm{O}_{2}$ treatment

The SNHG1-overexpressing vector (pcDNA3.1-SNHG1), the BCL2L2-overexpressing vector (pcDNA3.1BCL2L2) and the empty vector (pcDNA3.1) were obtained from the GenePharma (Shanghai, China). The miRNAs including miR-195 mimic, miR-195 inhibitor and the negative control (NC) miRNAs (mimic NC and inhibitor NC) were obtained from RiboBio (Guangzhou, China). The small interfering RNAs (siRNAs) for BCL2L2 and the scrambled siRNA (siNC), and the SNHG1 siRNA (si-SNHG1) and the scrambled siRNA for SNGH1 (siNC) were obtained from RiboBio. The cell transfection with plasmids, miRNAs or siRNAs was performed by using the Lipofectamine 2000 reagent (Invitrogen, Carlsbad, USA) according to the manufacturer's protocol. At $48 \mathrm{~h}$ after transfection, the cells were used for further experimentation. For the $\mathrm{H}_{2} \mathrm{O}_{2}$ treatment, cells were treated with $100 \mu \mathrm{M} \mathrm{H}_{2} \mathrm{O}_{2}$ for 6,12 and $24 \mathrm{~h}$, and after treatments, cells were processed for further experiment.

\section{Quantitative real-time PCR ( $q$ RT-PCR)}

Total RNA from HCMs was extracted by using Trizol reagent (Invitrogen) according to the manufacturer's protocol. MiR-195 was reversely transcribed by using miScript II RT kit (Qiagen, Valencia, USA) and quantified by qRT-PCR with the miScript SYBR green PCR Kit (Qiagen). SNHG1 and BCL2L2 mRNA were reversely transcribed to cDNA by using an iScript cDNA Synthesis Kit (Bio-Rad, Hercules, USA). The qRT-PCR was performed on an ABI 7500 thermocycler (Applied Biosystems, Foster City, USA). U6 was used as the internal control for miR-195, and GAPDH was used as the internal control for SNHG1 and BCL2L2. The relative expression of genes was calculated by using the comparative Ct method.

\section{Cell viability assay}

The cell viability was detected by MTT assay. Briefly, cells (5000 cells/well) were seeded in the 96-well plates. At $80 \%$ confluence, cells were received different treatments, and then the cells were incubated with $20 \mu \mathrm{l}$ of MTT at room temperature for $4 \mathrm{~h}$. After removing the medium, the blue formazan was dissolved with $200 \mu \mathrm{l}$ dimethyl sulfoxide (Sigma, St. Louis, USA), and the cell viability was revealed by detecting the absorbance at $550 \mathrm{~nm}$.

\section{Flow cytometry}

Cell apoptosis was detected by flow cytometry by using propidium iodide (PI) and fluorescein isothiocynate (FITC)-conjugated Annexin V staining. Briefly, treated cells were washed in phosphate buffered saline and fixed in 70\% ethanol. Fixed cells were then washed with phosphate buffered saline and incubated with PI/FITC-Annexin V in the presence of $50 \mu \mathrm{g} / \mathrm{ml}$ RNase A (Sigma) at room temperature in the dark. Flow cytometry analysis was performed by using the FACScan flow cytometer (Beckman Coulter, Fullerton, USA), and cell apoptotic rate was analyzed by FlowJo software (Tree Star, San Carlos, USA).

\section{Caspase-3 activity}

The caspase- 3 activity was detected by the caspase- 3 activity kit. The caspase- 3 was detected by determining its activity using an Apo-ONE Homogeneous Caspase-3/7 Assay Kit from Promega (Madison, USA) according to the manufacturer's protocol.

\section{Luciferase reporter assay}

The SNHG1 and the 3' 3'UTR of BCL2L2 were amplified by PCR, and the amplified PCR products were ligated into the pGL3 reporter vector (Promega) immediately downstream of the stop codon of the luciferase gene. The mutant SNHG1 and the mutant 3'UTR of BCL2L2 were generated by using the QuickChange II XL site-directed mutagenesis kit (Stratagene, San Diego, USA). For the measurement of the luciferase activity, cells were cultured in 24-well plates and were co-transfected with different miRNAs and luciferase constructs of pGL3-SNHG1-WT, pGL3-SNHG1-MUT, pGL3-BCL2L2 3'UTR-WT, or pGL3-BCL2L2 3'UTR-MUT. The Renilla luciferase plasmid was also co-transfected as an internal control. At $48 \mathrm{~h}$ after transfection, cells were lysed and the luciferase activity was detected by using the Dual Luciferase Reporter Assay kit (Promega) according to the manufacturer's protocol. 
Western blot

Proteins were extracted from the HCMs by using the RIPA buffer supplemented with a protease inhibitor cocktail (Sigma). The proteins were separated on 10\% SDS-PAGE gel and then transferred to the PVDF membrane (Millipore, Billerica, USA). The membranes were then blocked with 5\% skimmed milk followed by incubating with the following antibodies including anti-BCL2L2 (1:1500), anti-cleaved caspase-3 (1:1000), anti-cleaved caspase-9 (1:1500), anti-Bax (1:1000) and anti- $\beta$-actin (1:2000; Abcam, Cambridge, USA) at $4^{\circ} \mathrm{C}$ overnight. After overnight incubation, the membranes were then incubated with secondary antibodies conjugated with horseradish peroxidase for $1 \mathrm{~h}$ at room temperature, and immunodetection was perfomed by using the ECL kit (Millipore) according to manufacturer's protocol. $\beta$-actin was used as an internal control.

\section{Statistical analysis}

All the statistical analysis was performed by using the GraphPad Prism software Version 6.0. All the data were presented as mean \pm standard deviation. Differences between groups were compared by Student's t-test or one-way ANOVA followed by Bonferroni's multiple comparison test. $\mathrm{P}<0.05$ was considered statistically significant.

\section{Results}

Hydrogen peroxide $\left(\mathrm{H}_{2} \mathrm{O}_{2}\right)$ treatment suppressed cell viability, induced cell apoptosis and suppressed SNHG1 expression in HCMS

$\mathrm{H}_{2} \mathrm{O}_{2}$ is well known for its role of inducing cell death, and we examined the effects of $\mathrm{H}_{2} \mathrm{O}_{2}$ on cell viability, cell apoptosis and SNHG1 expression in HCMs. The HCMs were treated with $100 \mu \mathrm{M} \mathrm{H}_{2} \mathrm{O}_{2}$ for 6,12 and $24 \mathrm{~h}$, respectively, and $\mathrm{H}_{2} \mathrm{O}_{2}$ treatment time-dependently suppressed the cell viability (Fig. 1A), increased the cell apoptotic rate (Fig. 1B) and the caspase-3 activity (Fig. 1C) of HCMs. In addition, qRT-PCR analysis showed that $\mathrm{H}_{2} \mathrm{O}_{2}$ treatment time-dependently suppressed the expression levels of SNHG1 in HCMs (Fig. 1D), suggesting the involvement of SNHG1 in the $\mathrm{H}_{2} \mathrm{O}_{2}$-mediated effects in HCMs.

Fig. 1. The effects of $\mathrm{H}_{2} \mathrm{O}_{2}$ treatment on cell viability, cell apoptosis and SNHG1 expression levels in HCMs. (A) The cell viability of HCMs upon $\mathrm{H}_{2} \mathrm{O}_{2}$ treatment. HCMs were treated with $100 \mu \mathrm{M} \mathrm{H}_{2} \mathrm{O}_{2}$ for 6, 12 and $24 \mathrm{~h}$, respectively, and cell viability was detected by MTT assay. (B) The cell apoptosis of HCMs upon $\mathrm{H}_{2} \mathrm{O}_{2}$ treatment. HCMs were treated with $100 \mu \mathrm{M} \mathrm{H}_{2} \mathrm{O}_{2}$ for 6, 12 and $24 \mathrm{~h}$, respectively, and cell apoptosis was detected by flow cytometry. (C) The caspase-3 activity of HCMs upon $100 \mu \mathrm{M} \mathrm{H}_{2} \mathrm{O}_{2}$ treatment for 6,12 and $24 \mathrm{~h}$,
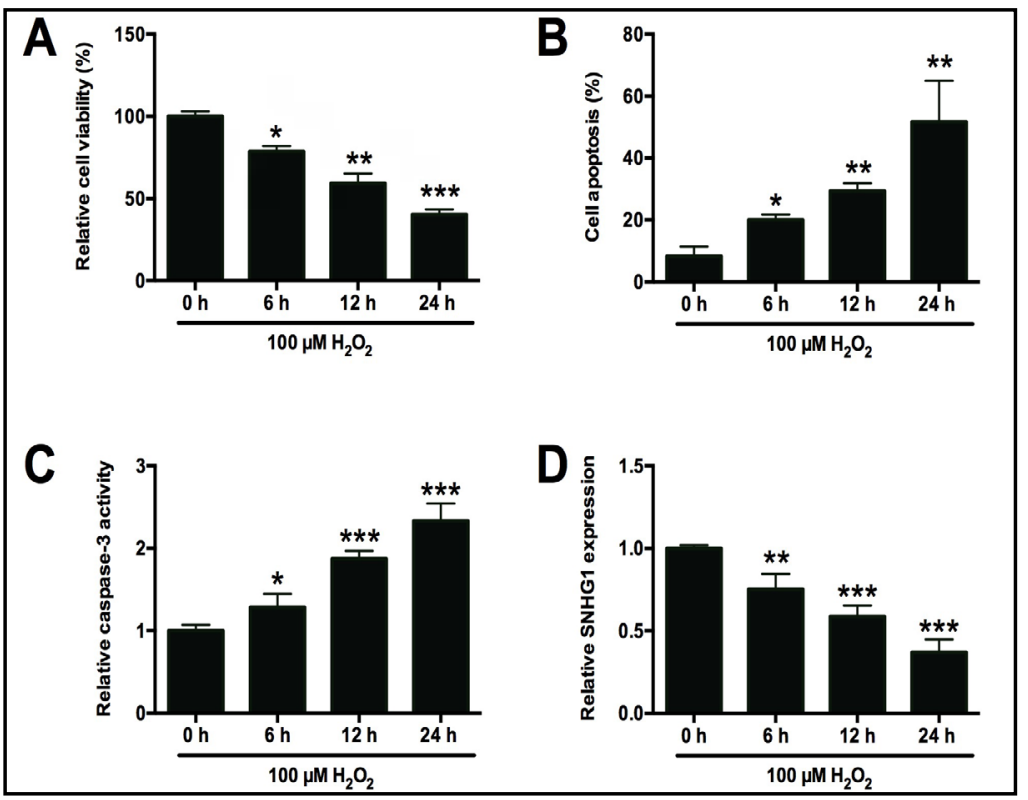

respectively. (D) The SNHG1 expression levels in cardiomyocytes upon $\mathrm{H}_{2} \mathrm{O}_{2}$ treatment. HCMs were treated with $100 \mu \mathrm{M} \mathrm{H}_{2} \mathrm{O}_{2}$ for 6, 12 and $24 \mathrm{~h}$, respectively, and the SNHG1 expression levels were detected by qRTPCR assay. $\mathrm{N}=3 ;{ }^{*} \mathrm{P}<0.05,{ }^{* *} \mathrm{P}<0.01$ and ${ }^{* * *} \mathrm{P}<0.001$ versus control group ( 0 h group). 
Fig. 2. The effects of SNHG1 overexpression on cell viability, cell apoptosis in $\mathrm{H}_{2} \mathrm{O}_{2}$-treated HCMs. (A) The SNGH1 expression levels in cardiomyocytes after transfecting with pcDNA3.1 or pcDNA3.1-SNGH1 were determined by qRT-PCR assay. Overexpression of SNHG1 attenuated the effects of $\mathrm{H}_{2} \mathrm{O}_{2}$ on (B) cell viability, (C) cell apoptosis and (D) caspase-3 activity in HCMs. HCMs were transfected with SNHG1overexpressing vector (SNHG1 group) or empty vector (pcDNA3.1). At 48
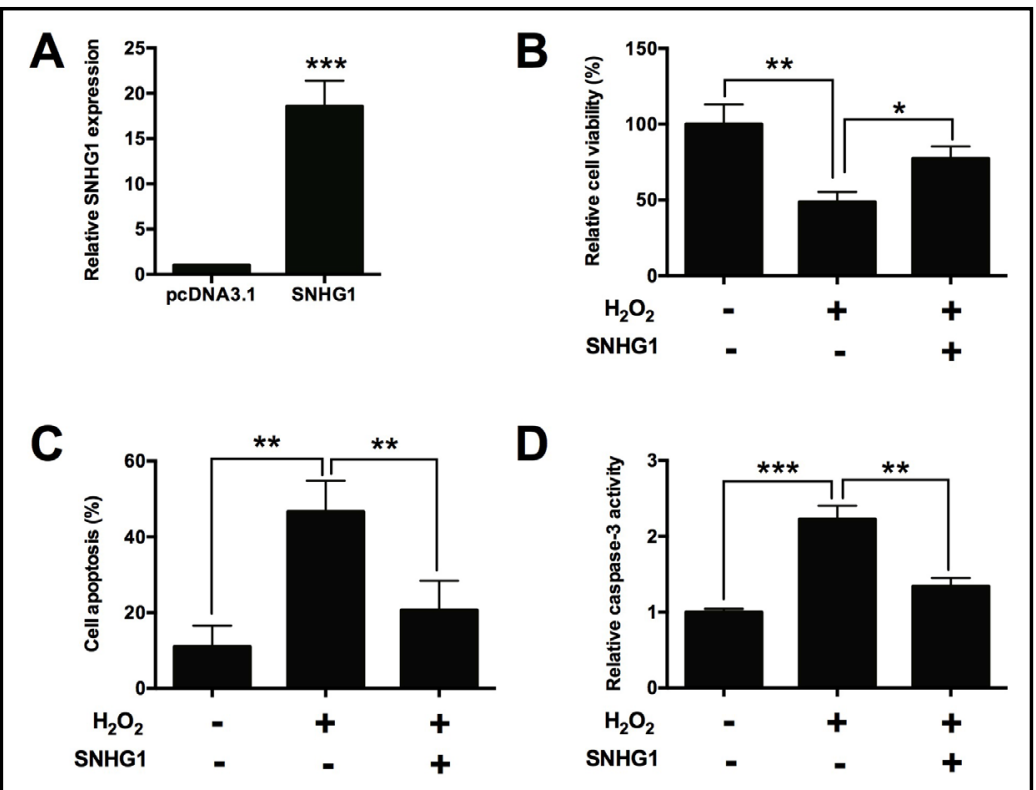
$\mathrm{h}$ after transfection, the HCMs were treated with $100 \mu \mathrm{M} \mathrm{H}_{2} \mathrm{O}_{2}$ for $24 \mathrm{~h}$, and the cell viability, cell apoptosis and caspase-3 activity of cardiomyocytes were detected by MTT assay, flow cytometry and caspase- 3 activity assay, respectively. $\mathrm{N}=$ 3 ; significant differences between groups were shown as ${ }^{*} \mathrm{P}<0.05,{ }^{* *} \mathrm{P}<0.01$ and ${ }^{* * *} \mathrm{P}<0.001$.

The effects of SNHG1 on cell viability, cell apoptosis in H2O2-treated HCMS

To explore the effects of SNHG1 on cell viability and cell apoptosis, the SNHG1overexpressing vector was constructed. As shown in Fig. 2A, transfecting with SNHG1overexpressing vector in HCMs increased the SNHG1 expression level by more than 15 fold compared with control group. Enhanced expression of SNHG1 attenuated the inhibitory effects of $\mathrm{H}_{2} \mathrm{O}_{2}$ on cell viability of $\mathrm{HCMs}$ as revealed by MTT assay (Fig. 2B). Flow cytometry and caspase-3 activity assay further revealed that enhanced expression of SNHG1 attenuated the $\mathrm{H}_{2} \mathrm{O}_{2}$-induced increase in cell apoptotic rate and caspase-3 activity of HCMs (Fig. 2C and 2D). Furthermore, the knockdown effects of SNGH1 on cell viability and cell apoptosis in $\mathrm{H}_{2} \mathrm{O}_{2}$ treated HCMs were also determined. Transfection with si-SNHG1 significantly suppressed the expression level of SNHG1 in HCMs compared with siNC group (Fig. 3A). MTT assay showed that knockdown of SNHG1 further suppressed cell viability of $\mathrm{H}_{2} \mathrm{O}_{2}$-treated HCMs (Fig. 3B). In addition, flow cytometry and caspase-3 activity assay demonstrated that SNGH1 knockdown further enhanced the effects of $\mathrm{H}_{2} \mathrm{O}_{2}$ treatment on cell apoptosis of HCMs (Fig. $3 \mathrm{C}$ and 3D).

\section{MiR-195 is a target of SNHG1}

To further explore the molecular mechanism of SNHG1-mediated cell viability and apoptosis, we researched the potential miRNAs that can bind SNHG1. We employed the DIANA TOOLS program and found that SNHG1 harbors a miR-195 binding site (Fig. 4A), and we then constructed the luciferase constructs of SNHG1 (pGL3-SNHG1-WT) and its mutant form (pGL3-SNHG1-MUT). Transfection with miR-195 mimic significantly suppressed the luciferase activity of pGL3-SNHG1-WT constructs compared to mimic NC group (Fig. 3B), and transfection with miR-195 inhibitor increased the luciferase activity of pGL3-SNHG1WT constructs compared to inhibitor NC group in HEK293 cells (Fig. 4B). Transfection with miR-195 mimic or miR-195 inhibitor had no effect on the luciferase activity of pGL3-SNHG1MUT constructs in HEK293 cells (Fig. 4C). Transfection with SNHG1-overexpressing vector in HCMs suppressed the expression of miR-195 in HCMs compared with control group (Fig. 4D). 
Fig. 3. The effects of SNGH1 knockdown on cell viability, cell apoptosis in $\mathrm{H}_{2} \mathrm{O}_{2}$-treated $\mathrm{HCM}_{\mathrm{S}^{*}}$ (A) The SNGH1 expression levels in cardiomyocytes after transfecting with siNC or siSNHG1 were determined by qRT-PCR assay. Knockdown of SNHG1 enhanced the effects of (B) cell viability, (C) cell apoptosis and (D) caspase-3 activity in HCMs. HCMs were transfected with scrambled siRNA (siNC) or SNHG1 siRNA (si-SNGH1). At $48 \mathrm{~h}$ after transfection, HCMs were treated with $100 \mu \mathrm{M}$ $\mathrm{H}_{2} \mathrm{O}_{2}$ for $24 \mathrm{~h}$, and the

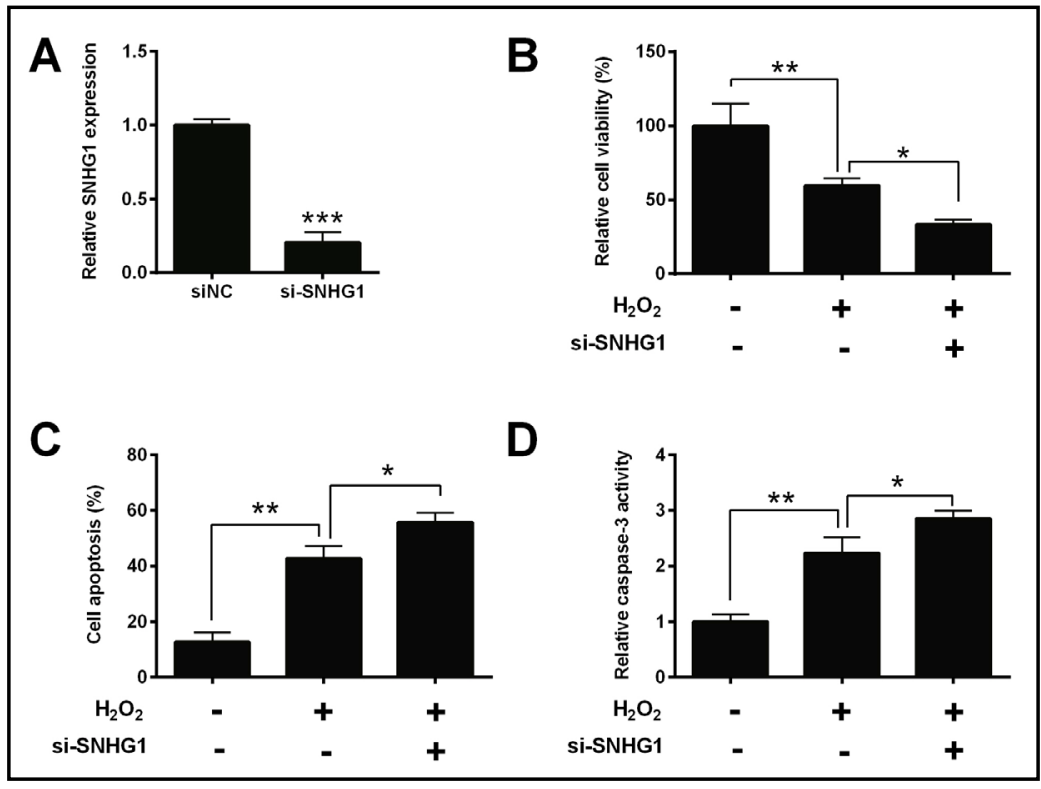
cell viability, cell apoptosis and caspase- 3 activity of cardiomyocytes were detected by MTT assay, flow cytometry and caspase-3 activity assay, respectively. $\mathrm{N}=3$; Significant differences between groups were shown as $* \mathrm{P}<0.05$, ${ }^{* *} \mathrm{P}<0.01$ and ${ }^{* * *} \mathrm{P}<0.001$.

Fig. 4. MiR-195 is a target of SNHG1. (A) Sequence complementarity between miR195 and SNHG1 (wild type, WT; mutant, MUT). (B) HEK293 cells were co-transfected with the luciferase constructs of pGL3SNGH1-WT and mimic NC, miR195 mimic, inhibitor NC or miR195 inhibitor, and luciferase activity was analyzed at $48 \mathrm{~h}$ post transfection. (C) HEK293 cells were co-transfected with the luciferase constructs of pGL3-SNGH1-MUT and mimic NC, miR-195 mimic, inhibitor NC or miR-195 inhibitor, and luciferase activity was analyzed at $48 \mathrm{~h}$ post transfection. (D) Overexpression of SNHG1 suppressed the expression of miR-195 in HCMs. HCMs were transfected with SNHG1overexpressing vector (SNHG1 group) or empty vector (pcDNA3.1). At $48 \mathrm{~h}$ after

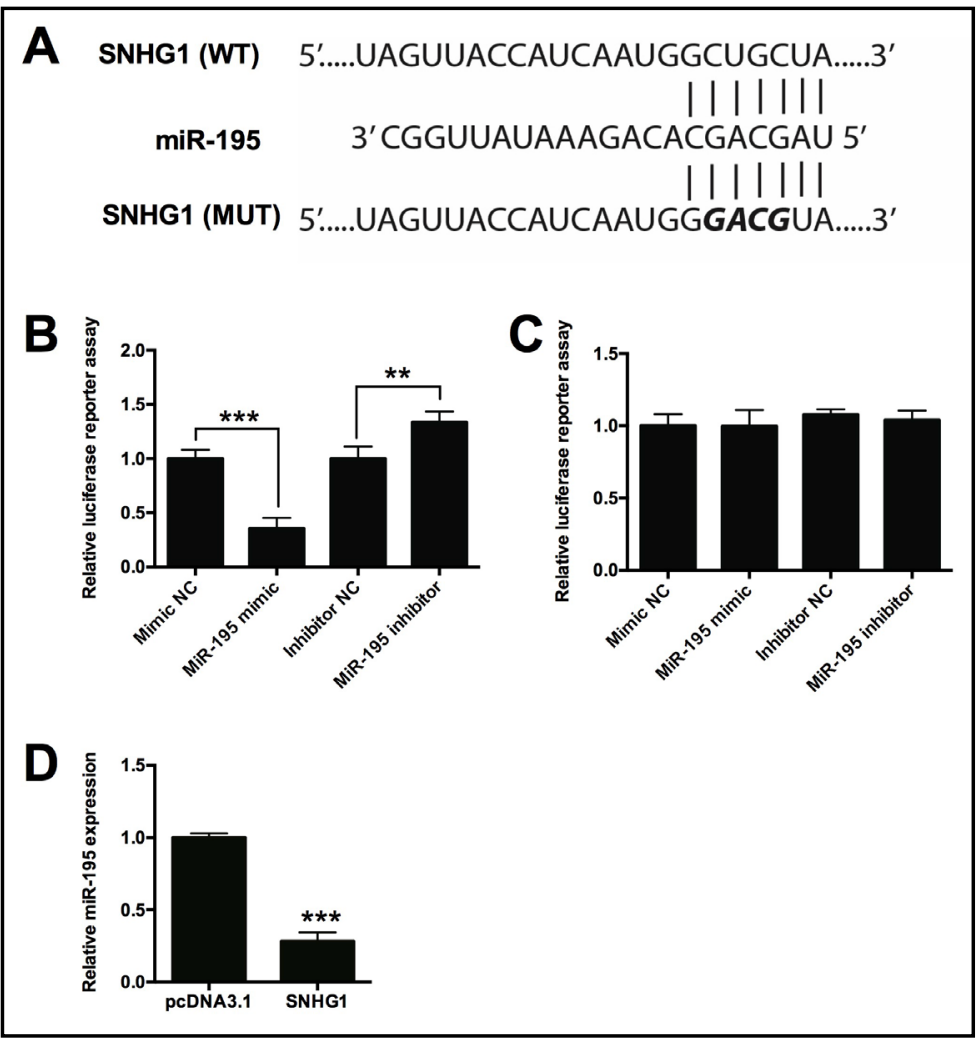
transfection, the expression level of miR-195 in HCMs was detected by qRT-PCR. N = 3; significant differences between groups were shown as ${ }^{* *} \mathrm{P}<0.01$ and ${ }^{* * *} \mathrm{P}<0.001$. 
Fig. 5. BCL2L2 is a target of miR195. (A) Sequence complementarity between miR-195 and BCL2L2 (wild type, WT; mutant, MUT). (B) HEK293 cells were cotransfected with the luciferase constructs of p G L 3 - B C L 2 L 2 3'UTR-WT and mimic NC, miR195 mimic, inhibitor NC or miR-195 inhibitor, and luciferase activity was analyzed at $48 \mathrm{~h}$ post transfection. (C) HEK293 cells were cotransfected with the luciferase constructs of p G L 3 - B C L 2 L 2 3' U T R - M U T and mimic NC, miR-195 mimic, inhibitor NC or miR-195 inhibitor, and luciferase activity was analyzed at $48 \mathrm{~h}$ post transfection. Overexpression of miR-195 suppressed the (D) mRNA and (E) protein expression levels of BCL2L2 in HCMs. HCMs were transfected with mimic NC or miR-195 mimic. At $48 \mathrm{~h}$ after transfection, the mRNA and protein levels of BCL2L2 in HCMs were detected by qRT-PCR and western blot, respectively. Overexpression of SNHG5 increased the (F) mRNA and (G) protein levels of BCL2L2 in HCMs. HCMs were transfected with SNGH1-overexpressing vector (SNHG1 group) or empty vector (pcDNA3.1 group). At $48 \mathrm{~h}$ after transfection, the mRNA and protein levels of BCL2L2 in HCMs were detected by qRT-PCR and western blot, respectively. Overexpression of BCL2L2 increased the (H) mRNA and (I) protein levels of BCL2L2 in HCMs. HCMs were transfected with BCL2L2-overexpression vector (BCL2L2 group) or empty vector (pcDNA3.1 group). Overexpression of SNHG1 attenuated the effects of miR-195 on (J) cell viability, (K) cell apoptosis and (L) caspase-3 activity in HCMs. HCMs were co-transfected with mimic NC + pcDNA3.1, miR-195 mimic + pcDNA3.1 or miR-195 mimic + pcDNA3.1-BCL2L2. At $48 \mathrm{~h}$ after transfection, the cell viability, cell apoptosis and caspase- 3 activity of HCMs were detected MTT assay, flow cytometry and caspase-3 activity assay, respectively. $\mathrm{N}=3$; significant differences between groups were shown as ${ }^{*} \mathrm{P}<0.05,{ }^{* *} \mathrm{P}<0.01$ and ${ }^{* * *} \mathrm{P}<0.001$.
A bCL2l2 3'Utr (WT) 5'....GUGCAUUAUGCACUUGCUGCUG......3'

miR-195 ACGACGAU 5'

BCL2L2 3'UTR (MUT) 5'....GUGCAUUAUGCACUUCGACCUG......3'

B

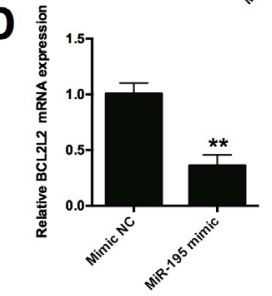

E

G
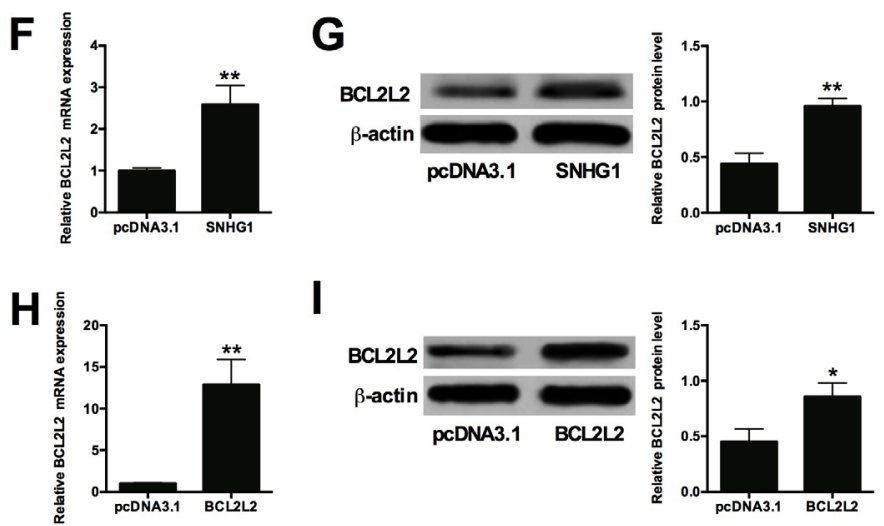

I
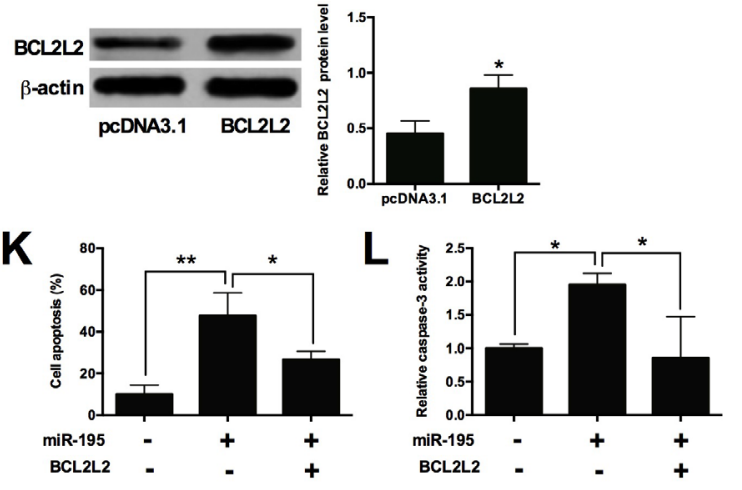
BCL2L2 is a target of miR-195

As miRNAs regulate the gene expression via targeting the 3'UTR, and we performed further bioinformatics analysis by using the TargetScan tool and found that BCL2L2 was one of the potential targets of miR-195 (Fig. 5A), and BCL2L2 was selected for further examination due to its important role in the regulation of apoptosis. We constructed the luciferase constructs of BCL2L2 3'UTR (pGL3-BCL2L2 3'UTR-WT) and its mutant form (pGL3-BCL2L2 3'UTR-MUT). Transfection with miR-195 mimic significantly suppressed the luciferase activity of pGL3-BCL2L2 3'UTR-WT constructs compared to mimic NC group (Fig. 5B), and transfection with miR-195 inhibitor increased the luciferase activity of pGL3BCL2L2 3'UTR-WT constructs compared to inhibitor NC group in HEK293 cells (Fig. 5B). Transfection with miR-195 mimic or miR-195 inhibitor had no effect on the luciferase activity of pGL3-BCL2L2 3'UTR-MUT constructs in HEK293 cells (Fig. 5C). Transfection with miR195 mimic also suppressed the mRNA and protein expression levels of BCL2L2 in HCMs (Fig. 5D and 5E). In addition, transfection with SNHG1-overexpressing vector in cardiomyocytes increased the mRNA and protein expression levels of BCL2L2 in cardiomyocytes (Fig. 5F and 5G). Transfection with BCL2L2-overexpressing vector in cardiomyocytes increased the mRNA and protein expression levels of BCL2L2 in cardiomyocytes (Fig. 5H and 5I). The in vitro functional assays showed that overexpression of miR-195 inhibited the cell viability and increased cell apoptotic rate and caspase-3 activity of HCMs (Fig. 5J-5L), and enforced expression of BCL2L2 attenuated miR-195-mediated effects on cell viability, cell apoptotic rate and caspase-3 activity of HCMs (Fig. 5J-5L).

SNHG1 regulates cell viability and cell apoptosis via targeting miR-195 and BCL2L2 in HCMs

To explore whether SNHG1 regulates cell viability and apoptosis via miR-195 and BCL2L2 in cardiomyocytes, the cardiomyocytes were co-transfected with SNHG1-overexpressing vector and miR-195 mimic, and the results showed that miR-195 overexpression attenuated SNHG1-induced increase in cell viability and decrease in cell apoptotic rate and caspase- 3

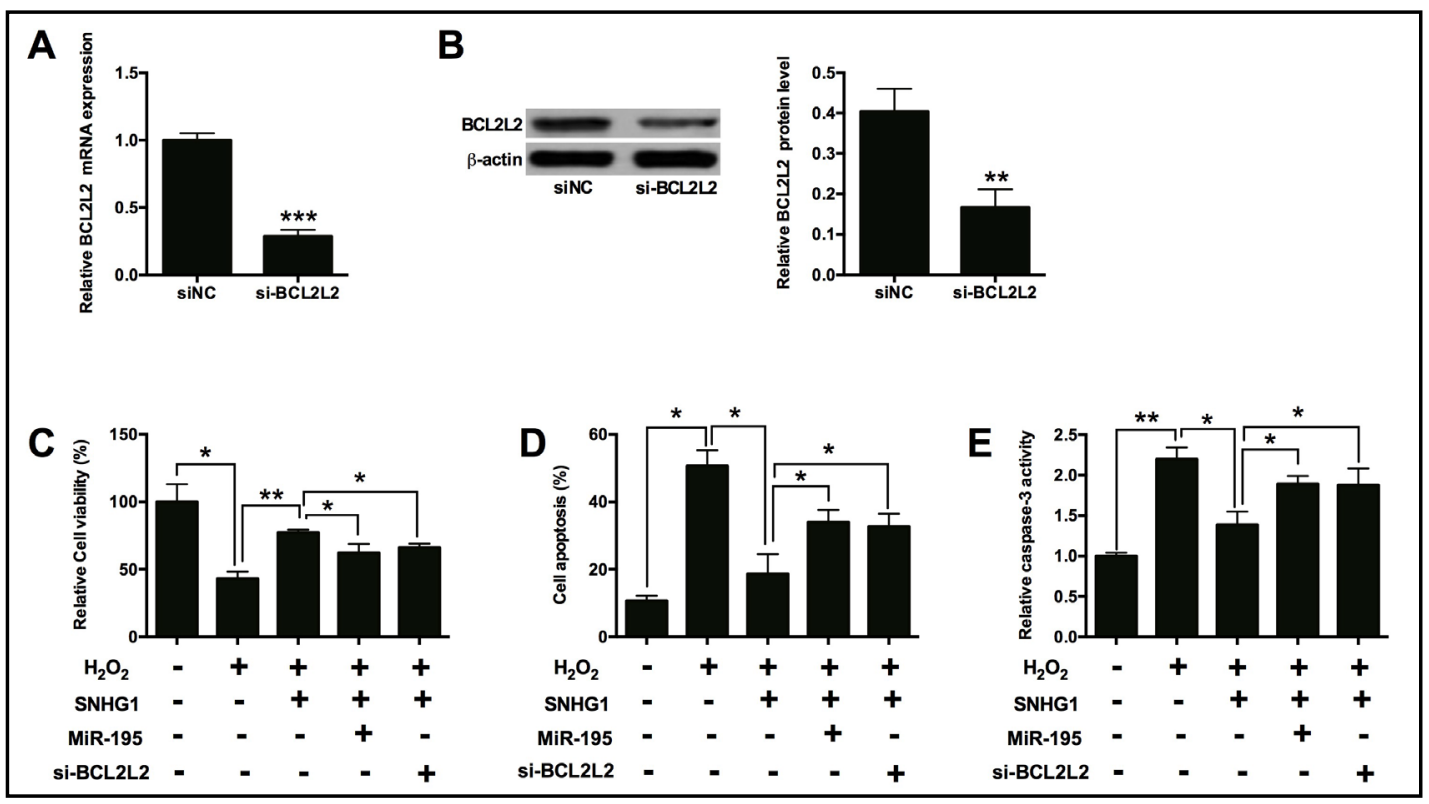

Fig. 6. SNHG1 affects cell viability and cell apoptosis via regulating miR-195 and BCL2L2 in HCMs. Knockdown of BCL2L2 decreased the (A) mRNA and (B) protein levels of BCL2L2 in HCMs. HCMs were transfected with BCL2L2 siRNA (si-BCL2L2 group) or scrambled siRNA (siNC group). MiR-195 overexpression or BCL2L2 knockdown attenuated the effects of SNHG1 overexpression on (C) cell viability, (D) cell apoptosis and (E) caspase-3 activity in $\mathrm{H}_{2} \mathrm{O}_{2}$-treated $\mathrm{HCMs} . \mathrm{N}=3$; significant differences between groups were shown as $* \mathrm{P}<0.05$ and ${ }^{* *} \mathrm{P}<0.01$ and ${ }^{* * *} \mathrm{P}<0.001$. 


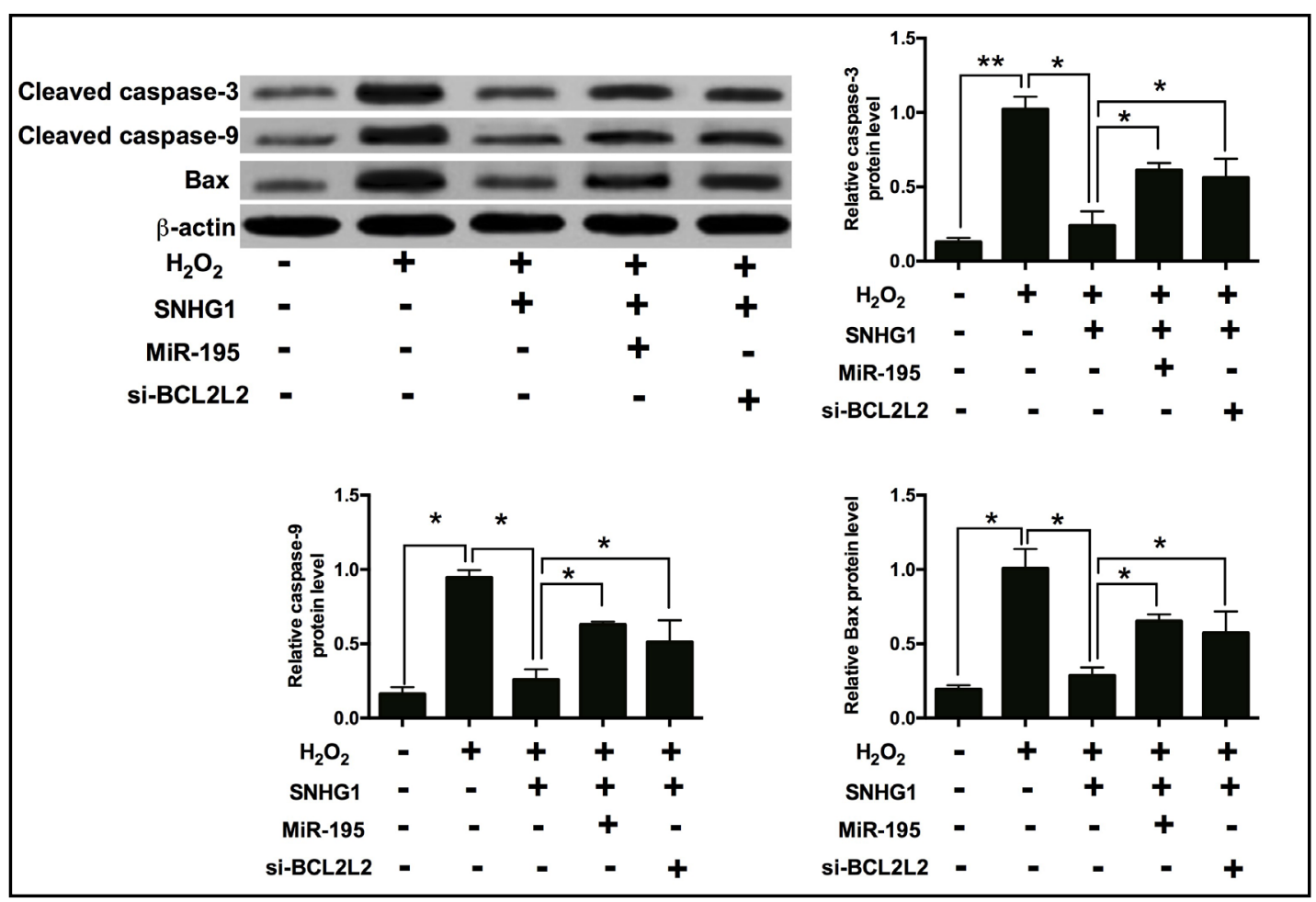

Fig. 7. SNHG1 affects the pro-apoptotic protein levels via regulating miR-195 and BCL2L2 in HCMs. MiR-195 overexpression or BCL2L2 knockdown attenuated the effects of SNGH1 overexpression on protein levels of cleaved caspase-3, cleaved caspase- 9 and Bax in $\mathrm{H}_{2} \mathrm{O}_{2}$-treated HCMs. $\mathrm{N}=3$; significant differences between groups were shown as $* \mathrm{P}<0.05$ and ${ }^{* *} \mathrm{P}<0.01$.

activity in $\mathrm{H}_{2} \mathrm{O}_{2}$-treated HCMs (Fig. 6C-6E). In addition, the HCMs were also co-transfected with SNHG1-overexpressing vector and BCL2L2 siRNA, and transfection with BCL2L2 siRNA in cardiomyocytes suppressed the mRNA and protein expression levels of BCL2L2 in cardiomyocytes (Fig. 6A and 6B). Similar results showed that BCL2L2 knockdown also attenuated the effects of SNHG1 overexpression on cell viability, cell apoptotic rate and caspase-3 activity in $\mathrm{H}_{2} \mathrm{O}_{2}$-treated HCMs (Fig. 6C-6E).

SNHG1 regulates the pro-apoptotic protein levels via targeting miR-195 and BCL2L2 in HCMS

To explore the molecular mechanism of SNHG1-mediated cell apoptosis in cardiomyocytes, we examined the protein levels of cleaved caspase-3, cleaved caspase- 9 and Bax. $\mathrm{H}_{2} \mathrm{O}_{2}$ treatment increased the protein levels of cleaved caspase- 3 , cleaved caspase- 9 and Bax in HCMs (Fig. 7), and SNHG1 overexpression attenuated the $\mathrm{H}_{2} \mathrm{O}_{2}$-inudced increased in the protein levels of cleaved caspase-3, cleaved caspase- 9 and Bax in the HCMs (Fig. 7). In addition, miR-195 overexpression or BCL2L2 knockdown attenuated the effects of SNHG1 overexpression on protein levels of cleaved caspase-3, cleaved caspase- 9 and Bax in $\mathrm{H}_{2} \mathrm{O}_{2}$ treated HCMs (Fig. 7).

\section{Discussion}

Ischemic heart disease is a big threat to the human heath worldwide, and studies have demonstrated that dysregulation of cardiomyocyte apoptosis contributed to the development of ischemia heart disease such as heart failure and myocardial infarction [7]. To understand the molecular mechanisms that regulate cardiomyocyte apoptosis is important 
for prevention and treatment of heart disease. Our results showed that SNHG1 expression was down-regulated upon $\mathrm{H}_{2} \mathrm{O}_{2}$ treatment, and overexpression of SNHG1 attenuated the effects of $\mathrm{H}_{2} \mathrm{O}_{2}$ treatment on cell viability and cell apoptosis in HCMs. SNHG1 was further found to function as a ceRNA to negatively regulate the expression of miR-195, which results in the regulation of BCL2L2 expression in the HCMs. Our study revealed a novel SNHG1/miR195/BCL2L2 signaling axis in the regulating of cardiomyocyte apoptosis.

$\mathrm{H}_{2} \mathrm{O}_{2}$ treatment is a potent inducer for cardiomyocyte apoptosis, which has been welldocumented in many studies [22]. In the present study, we consistently found that $\mathrm{H}_{2} \mathrm{O}_{2}$ treatment suppressed cell viability and increased cell apoptosis and caspase- 3 activity in the HCMs, and the effects of $\mathrm{H}_{2} \mathrm{O}_{2}$ treatment were attenuated by SNHG1 overexpression. In the cancer studies, overexpression of SNHG1 enhanced cell viability, and inhibited apoptosis via regulating p53 and p53-targeted genes in the hepatocellular carcinoma cells [23]. SNHG1 was also found to be up-regulated in glioma and overexpression of SNHG1 enhanced cell proliferation and reduced cell apoptosis in the glioma cells [24]. In addition, SNHG1 can promote cell proliferation and inhibit apoptosis of lung cancer cells via the Tap63/ZEB1 pathway [25]. Collectively, these results may suggest that SNHG1 exerts enhanced effects on cell viability and inhibitory effects on cell apoptosis in the HCMs.

As many studies have suggested the ceRNA role of lncRNA, miR-195 was predicted to involve the interaction with SNHG1 by using the DIANA TOOL. SNHG1 negatively regulated the expression of miR-195 in the HCMs and overexpression of miR-195 suppressed cell viability and induced apoptosis in the HCMs. The role of miR-195 in regulation of cardiomyocyte apoptosis has been revealed in several studies. Zhu et al., showed that miR-195 promoted palmitate-induced apoptosis in cardiomyocytes via targeting Sirt1 [26]. MiR-195 expression was found to be up-regulated in ischemia/reperfusion injury, and miR-195 overexpression was shown to promote apoptosis by targeting Bcl-2 and inducing mitochondrial apoptotic pathway [27]. In addition, miR-195 was significantly increased upon hypoxia/reoxygenation injury and enhanced cardiomyocyte apoptosis via targeting c-myc [28]. Taken together, the effects of SNHG1 on cardiomyocyte apoptosis may be mediated via negatively regulating miR-195.

MiRNAs regulated the gene expression via targeting its 3'UTR, and BCL2L2 was predicted to be one of potential targets of miR-195, which was confirmed by luciferase reporter assay and in vitro functional assays. BCL2L2 belongs to the BLC-2 family that are important regulators of apoptosis and plays important roles in the occurrence and development of tumor $[29,30]$. In the cardiomyocytes, Long et al., showed that the lncRNA Five Prime to Xist regulated cardiomyocyte apoptosis via modulating the expression of BCL2L2 which was targeted by miR-29b-1-5p [31]. In our study, overexpression of miR-195 or BCL2L2 knockdown attenuated the effects of SNHG1 overexpression on cell viability, cell apoptosis as well as pro-apoptotic protein levels, suggesting that SNHG1 regulated cell viability and cell apoptosis via targeting miR-195 and BCL2L2 in the HCMs.

\section{Conclusion}

Our results suggested that overexpression of SNHG1 may play an anti-apoptotic role in HCMs apoptosis partly via sponging miR-195 and finally regulating the expression of BLC2L2. Further in vivo studies may be required to clarify the role of SNHG1 in the regulation of HCMs apoptosis-related heart diseases.

\section{Acknowledgements}

This study was supported by the General Program of Natural Science Foundation of Shaanxi Province (Project No. 2017JM8084). 


\section{Cellular Physiology Cell Physiol Biochem 2018;50:1029-1040 \begin{tabular}{l|l|l} 
DOI: 10.1159/000494514 & (c) 2018 The Author(s). Published by S. Karger AG, Basel
\end{tabular} Published online: 24 October 2018 www.karger.com/cpb}

Zhang et al.: SNHG1 and Cardiomyocyte Apoptosis

\section{Disclosure Statement}

The authors declare to have no competing interests.

\section{References}

1 Mitchell JD, Brown DL: Harmonizing the Paradigm With the Data in Stable Coronary Artery Disease: A Review and Viewpoint. J Am Heart Assoc 2017;6. pii: e007006.

-2 Dong Y, Liu C, Zhao Y, Ponnusamy M, Li P, Wang K: Role of noncoding RNAs in regulation of cardiac cell death and cardiovascular diseases. Cell Mol Life Sci 2017;10.1007/s00018-017-2640-8.

$>3$ Granger DN, Kvietys PR: Reperfusion therapy-What's with the obstructed, leaky and broken capillaries? Pathophysiology 2017;10.1016/j.pathophys.2017.09.003.

4 Krzywonos-Zawadzka A, Franczak A, Sawicki G, Wozniak M, Bil-Lula I: Multidrug prevention or therapy of ischemia-reperfusion injury of the heart-Mini-review. Environ Toxicol Pharmacol 2017;55:55-59.

5 Yu SY, Tang L, Zhou SH: Long Noncoding RNAs: New Players in Ischaemia-reperfusion Injury. Heart Lung Circ 2017;10.1016/j.hlc.2017.09.011.

6 Moe GW, Marin-Garcia J: Role of cell death in the progression of heart failure. Heart Fail Rev 2016;21:157167.

7 Xia P, Liu Y, Cheng Z: Signaling Pathways in Cardiac Myocyte Apoptosis. Biomed Res Int 2016;2016:9583268.

-8 Dangwal S, Schimmel K, Foinquinos A, Xiao K, Thum T: Noncoding RNAs in Heart Failure. Handb Exp Pharmacol 2017;243:423-445.

-9 Archer K, Broskova Z, Bayoumi AS, Teoh JP, Davila A, Tang Y, Su H, Kim IM: Long Non-Coding RNAs as Master Regulators in Cardiovascular Diseases. Int J Mol Sci 2015;16:23651-23667.

10 Piccoli MT, Gupta SK, Thum T: Noncoding RNAs as regulators of cardiomyocyte proliferation and death. J Mol Cell Cardiol 2015;89:59-67.

-11 Wang K, Long B, Zhou LY, Liu F, Zhou QY, Liu CY, Fan YY, Li PF: CARL lncRNA inhibits anoxia-induced mitochondrial fission and apoptosis in cardiomyocytes by impairing miR-539-dependent PHB2 downregulation. Nat Commun 2014;5:3596.

12 Wang K, Liu CY, Zhou LY, Wang JX, Wang M, Zhao B, Zhao WK, Xu SJ, Fan LH, Zhang XJ, Feng C, Wang CQ Zhao YF, Li PF: APF IncRNA regulates autophagy and myocardial infarction by targeting miR-188-3p. Nat Commun 2015;6:6779.

13 Zhang J, Gao C, Meng M, Tang H: Long Noncoding RNA MHRT Protects Cardiomyocytes against H2O2Induced Apoptosis. Biomol Ther (Seoul) 2016;24:19-24.

14 Liu Y, Yang Y, Li L, Liu Y, Geng P, Li G, Song H: LncRNA SNHG1 enhances cell proliferation, migration and invasion in cervical cancer. Biochem Cell Biol 2018;96:38-43.

15 Sun Y, Wei G, Luo H, Wu W, Skogerbo G, Luo J, Chen R: The long noncoding RNA SNHG1 promotes tumor growth through regulating transcription of both local and distal genes. Oncogene 2017;10.1038/ onc.2017.286.

16 Wang J, Cao L, Wu J, Wang Q: Long non-coding RNA SNHG1 regulates NOB1 expression by sponging miR326 and promotes tumorigenesis in osteosarcoma. Int J Oncol 2018;52:77-88.

-17 Tian L, Zheng F, Li Z, Wang H, Yuan H, Zhang X, Ma Z, Li X, Gao X, Wang B: miR-148a-3p regulates adipocyte and osteoblast differentiation by targeting lysine-specific demethylase 6b. Gene 2017;627:32-39.

18 Li X, Kong M, Jiang D, Qian J, Duan Q, Dong A: MicroRNA-150 aggravates H2O2-induced cardiac myocyte injury by down-regulating c-myb gene. Acta Biochim Biophys Sin (Shanghai) 2013;45:734-741.

19 Sun G, Lu Y, Li Y, Mao J, Zhang J, Jin Y, Li Y, Sun Y, Liu L, Li L: miR-19a protects cardiomyocytes from hypoxia/ reoxygenation-induced apoptosis via PTEN/PI3K/p-Akt pathway. Biosci Rep 2017;10.1042/bsr20170899.

20 Chen Z, Zhang S, Guo C, Li J, Sang W: Downregulation of miR-200c protects cardiomyocytes from hypoxiainduced apoptosis by targeting GATA-4. Int J Mol Med 2017;39:1589-1596.

21 Kang B, Li W, Xi W, Yi Y, Ciren Y, Shen H, Zhang Y, Jiang H, Xiao J, Wang Z: Hydrogen Sulfide Protects Cardiomyocytes against Apoptosis in Ischemia/Reperfusion through MiR-1-Regulated Histone Deacetylase 4 Pathway. Cell Physiol Biochem 2017;41:10-21. 


\section{Cellular Physiology Cell Physiol Biochem 2018;50:1029-1040 \begin{tabular}{l|l|l} 
DOI: 10.1159/000494514 & $\begin{array}{l}\text { O 2018 The Author(s). Published by S. Karger AG, Basel } \\
\text { www.karger.com/cpb }\end{array}$ \\
\hline
\end{tabular}}

Zhang et al.: SNHG1 and Cardiomyocyte Apoptosis

22 Zhang Y, Tocchetti CG, Krieg T, Moens AL: Oxidative and nitrosative stress in the maintenance of myocardial function. Free Radic Biol Med 2012;53:1531-1540.

-23 Zhang M, Wang W, Li T, Yu X, Zhu Y, Ding F, Li D, Yang T: Long noncoding RNA SNHG1 predicts a poor prognosis and promotes hepatocellular carcinoma tumorigenesis. Biomed Pharmacother 2016;80:73-79.

-24 Wang Q, Li Q Zhou P, Deng D, Xue L, Shao N, Peng Y, Zhi F: Upregulation of the long non-coding RNA SNHG1 predicts poor prognosis, promotes cell proliferation and invasion, and reduces apoptosis in glioma. Biomed Pharmacother 2017;91:906-911.

-25 Zhang HY, Yang W, Zheng FS, Wang YB, Lu JB: Long non-coding RNA SNHG1 regulates zinc finger E-box binding homeobox 1 expression by interacting with TAp63 and promotes cell metastasis and invasion in Lung squamous cell carcinoma. Biomed Pharmacother 2017;90:650-658.

26 Zhu H, Yang Y, Wang Y, Li J, Schiller PW, Peng T: MicroRNA-195 promotes palmitate-induced apoptosis in cardiomyocytes by down-regulating Sirt1. Cardiovasc Res 2011;92:75-84.

27 Gao CK, Liu H, Cui CJ, Liang ZG, Yao H, Tian Y: Roles of MicroRNA-195 in cardiomyocyte apoptosis induced by myocardial ischemia-reperfusion injury. J Genet 2016;95:99-108.

-28 Chen C, Jia KY, Zhang HL, Fu J: MiR-195 enhances cardiomyocyte apoptosis induced by hypoxia/ reoxygenation injury via downregulating c-myb. Eur Rev Med Pharmacol Sci 2016;20:3410-3416.

29 Lima RT, Busacca S, Almeida GM, Gaudino G, Fennell DA, Vasconcelos MH: MicroRNA regulation of core apoptosis pathways in cancer. Eur J Cancer 2011;47:163-174.

-30 Petros AM, Olejniczak ET, Fesik SW: Structural biology of the Bcl-2 family of proteins. Biochim Biophys Acta 2004;1644:83-94.

31 Long B, Li N, Xu XX, Li XX, Xu XJ, Guo D, Zhang D, Wu ZH, Zhang SY: Long noncoding RNA FTX regulates cardiomyocyte apoptosis by targeting miR-29b-1-5p and Bcl2l2. Biochem Biophys Res Commun 2018;495:312-318. 